\title{
Artificial trans-acting small interfering RNA: a tool for plant biology study and crop improvements
}

\author{
Zhanyuan J. Zhang
}

Received: 24 December 2013 / Accepted: 5 March 2014 / Published online: 19 March 2014

(C) Springer-Verlag Berlin Heidelberg 2014

\begin{abstract}
Completion of whole genome sequencing in many plant species including economically important crop species not only opens up new opportunities but also imposes challenges for plant science research community. Functional validation and utilization of these enormous DNA sequences necessitate new or improved tools with high accuracy and efficiency. Of various tools, small RNAmediated gene silencing platform plays an important and unique role in functional verification of plant genes and trait improvements. Artificial trans-acting small interfering RNA (atasiRNA) has emerged as a potent and specific gene silencing platform which overcomes major limitations of other small RNA silencing approaches including doublestranded RNA, artificial microRNA (amiRNA), and microRNA-induced gene silencing. To best utilize atasiRNA platform, it is essential to be able to test candidate atasiRNAs efficiently through either in vivo or in vitro validation approach. Very recently, a breakthrough has been made in developing a new method for in vitro screen of amiRNA candidates, named "epitope-tagged protein-based amiRNA screens". Such a screen can be readily employed to validate atasiRNA candidates and thus accelerate the deployment of atasiRNA technology. Therefore, atasiRNA as an emerging tool shall accelerate both plant biology study and crop genetic improvements including trait stacking.
\end{abstract}

Keywords Artificial trans-acting small interfering RNA . amiRNA $\cdot$ siRNA $\cdot$ dsRNA $\cdot$ Plant

Z. J. Zhang $(\triangle)$

Plant Transformation Core Facility, Division of Plant Sciences, University of Missouri, 1-33 Agriculture Building, Columbia, MO 65211, USA

e-mail: zhangzh@missouri.edu

$\begin{array}{ll}\text { Abbreviations } & \\ \text { AGO } & \text { Argonaute protein } \\ \text { amiRNA } & \text { Artificial microRNA } \\ \text { atasiRNA } & \text { Artificial trans-acting small interfering } \\ & \text { RNA } \\ \text { CRISPR-Cas } & \text { Clustered regularly interspaced short palin- } \\ & \text { dromic repeats } \\ \text { DCL } & \text { Dicer-like protein } \\ \text { dsRNA } & \text { Double-stranded RNA } \\ \text { ETPamir } & \text { Epitope-tagged protein-based amiRNA } \\ \text { GM } & \text { Genetically modified } \\ \text { hpRNA } & \text { Hairpin RNA } \\ \text { IR } & \text { Inverted repeat } \\ \text { MIGS } & \text { microRNA-induced gene silencing } \\ \text { RDR } & \text { RNA-dependent RNA polymerase } \\ \text { RNA } & \text { Pol II RNA polymerase II } \\ \text { RISC } & \text { RNA-induced silencing complex } \\ \text { TALEN } & \text { Transcription activator-like effector } \\ & \text { nuclease } \\ \text { TAS } & \text { Trans-acting siRNA locus } \\ \text { siRNA } & \text { Small interfering RNA } \\ \text { ZFN } & \text { Zinc finger nuclease }\end{array}$

\section{Introduction}

With the advent of post-genome era, the whole genome sequences of many plant species have now become available (http://en.wikipedia.org/wiki/List_of_sequenced_plant_ genomes; Feuillet et al. 2011; Bevan and Uauy 2013). Next and urgent tasks will be the functional verification of a large number of genes to accelerate plant biology study and crop genetic improvements. Disruption of a gene function using reverse genetics is a very important and relatively simple way to analyze gene functions and achieve crop 
trait improvements (Page and Grossniklaus 2002; Kumar et al. 2012). Recently, a plethora of creative ways has been devised to disrupt gene functions and screen mutations that are useful for gene functional analyses or for crop trait improvements (Yin and Zhang 2010). Of these various tools, RNA interference (RNAi) (Fire et al. 1998) has been employed as a universal platform for gene silencing in various organisms including plants (Waterhouse and Helliwell 2003; Shafran et al. 2008; Bellés 2010). Reverse genetics through RNAi is unique in its ability to directly correlate the target genes with their functions by loss-of-function. Verification of a gene function is difficult with conventional approaches such as biochemical assays and use of mutants. In particular, gene functional redundancy is an acute problem for functional verification using single gene mutation lines. In addition to plant biology studies, RNAi plays important and unique roles in crop genetic improvements (Auer and Frederick 2009; Baykal and Zhang 2010). RNA interference may achieve more significant crop improvements than conventional breeding, as illustrated by various important crop species with different improved traits (Liu et al. 2002; Ogita et al. 2003; Gilissen et al. 2005; Le et al. 2006; Bonfim et al. 2007; Shi et al. 2007; Dodo et al. 2008; Eady et al. 2008; Flores et al. 2008; Shimizu et al. 2009). Many of these studies show that the RNAi-mediated silencing is inheritable, alleviating long-standing concerns about the RNAi inheritability.

Classification, biogenesis, and functions of small regulatory RNAs: siRNA, miRNA, and tasiRNA

RNA interference is mediated by small RNAs-non-protein coding which can be categorized into two major classes: small interfering RNA (siRNA) and microRNA (miRNA) (Brodersen and Voinnet 2006; Jones-Rhoades et al. 2006; Vaucheret 2006; Siomi and Siomi 2009). In plants, siRNAs originate from invading viruses, transgene inverted repeats, centromeres, transposons, and other repetitive sequences. The long double-stranded RNA (dsRNA) from a transgene construct is a common entry transcript in siRNA pathway. There are three key steps in the siRNA biogenesis in plants. First, dsRNAs are diced into short (21-24 bp) siRNAs by RNAse-III Dicer-like 3 or 4 . The RNA-dependent RNA polymerase (RDR) is required to generate the long double-stranded RNAs if the dsRNAs are derived from single-stranded RNA. Second, the siRNA duplex is unwound and one strand is preferentially loaded to RNA-induced silencing complex (RISC). Third, the RISC finds its transcript targets, and the loaded single-stranded siRNA (guide strand) directs the sequence-specific cleavage or translational repression of the target transcript through the Argonaute protein (AGO) in the RISC. The siRNA is thought to be an RNA-based immune mechanism to fight off nucleic acid invaders or to maintain genome stability. In contrast, plant miRNAs are endogenous and originated from genome regions, normally intergenic regions or introns, as primary precursors (pri-miRNAs) transcribed by RNA polymerase II (Chen 2009). These precursors contain stem-loop structures, which harbor 21-nucleotide (nt) miRNA in $5^{\prime}$ or $3^{\prime}$ half of the stem. RNAse-III Dicer-like 1 (DCL1) then generates 21-nt miRNA/miRNA* duplex. Subsequently, the guide strand miRNA is preferentially loaded onto the AGO in the RISC while the miRNA* (passenger strand) is destroyed. The miRNA then directs the AGO in the RISC to cleave or translationally repress mRNA targets. The roles of miRNAs are to regulate plant developments and various physiological responses. Regardless of siRNA or miRNA/miRNA* duplex, they are all first $3^{\prime}$-end methylated by HUA ENHANCER 1 (HEN1) and the preferential loading of the guide strand is determined by thermodynamic asymmetry along the siRNA or miRNA/miRNA* duplex. The characteristic thermodynamic unstable $5^{\prime}$ end of the siRNA or miRNA strand allows this molecule to be loaded onto the RISC. Recently, plant siRNA- or miRNA-mediated translational repression of mRNA targets was found to be broadly applied (Brodersen et al. 2008). Intriguingly and importantly, the sequence complementarity between plant siRNA and miRNA, and its mRNA targets do not determine cleavage or translational repression of mRNA targets. This is in sharp contrast to animal models in which a complete or near-complete sequence complementarity causes mRNA target cleavage, while a low degree of complementarity causes translational inhibition of mRNA target. The mechanism that dictates cleavage or translational inhibition of mRNA targets in plants is still poorly understood.

One subclass of small regulatory RNAs is the transacting small interfering RNA (tasiRNA), which silences its mRNA target in trans (Chapman and Carrington 2007). The tasiRNA transcripts are generated from non-protein coding locus of plant genome region, i.e., trans-acting siRNA locus (TAS). To date four TAS loci have been found in plants: TAS1, TAS2, TAS3, and TAS4 (Peragine et al. 2004; Vazquez et al. 2004; Allen et al. 2005; Gasciolli et al. 2005; Xie et al. 2005; Yoshikawa et al. 2005; Rajagopalan et al. 2006). Of these, TAS1, TAS2 and TAS4 are found only in Arabidopsis thaliana: TAS1 and TAS2 each contains miR173 complementary site whereas TAS4 carries miR828-binding site (close to the $5^{\prime}$-end). TAS3 is universal in the plant kingdom and contains two miR390 complementary sites, one close to $5^{\prime}$ end, and the other close to $3^{\prime}$ end. These TAS miRNA-binding sites function as trigger sequences for endogenous miR173, miR390 or miR828 to trigger the degradation of the TAS transcript, producing phased 21-nt siRNAs. In TAS1, TAS2, and TAS4, the cleavage sites for generating phased siRNAs are located 
downstream of miRNA complementary sites, whereas in TAS3 the phased siRNAs are generated from the upstream sequence of the $3^{\prime}$-end miR390 complementary site. The Arabidopsis TAS1 has three family members (TASIa, $T A S 1 b$, and $T A S 1 c$ ). Thus far no additional family members have been found in TAS2, TAS3, or TAS4.

Figure 1 illustrates small regulatory RNA pathways including dsRNA, miRNA, and tasiRNA pathways.

Artificial tasiRNA: an emerging tool for plant biology studies and crop genetic improvements

The silencing induced by the dsRNA of a transgene construct has several significant limitations. In a dsRNA construct, each inverted repeat (IR) that forms dsRNA normally needs to be 300-800 bp long for effective RNAi (Watson et al. 2005). Such a dsRNA can produce many different siRNA species and a much higher chance of causing "off-target" effect (silencing of unintended gene) (Jackson et al. 2003) or "transitive silencing" (spreading of silencing to regions outside the inducer sequence) (Baulcombe 2007). Both can cause epigenetic modifications of cells in untended tissues due to the siRNA mobility, even if a transgene expression is controlled in a tissue-specific manner (Dunoyer et al. 2010; Martienssen 2010; Molnar et al. 2010). It is also difficult for dsRNA to silence a specific gene family member because of the high degree of sequence similarities. The dsRNA is also challenging for stacking multiple transgene cassettes in a complex metabolic engineering study or crop trait stacking. Such a study not only faces technical challenges in cloning multiple transgene cassettes, but is also restricted by promoter choices to avoid promoter homology-dependent gene silencing (Meyer and Saedler 1996). In addition, because the effect of long inverted repeat is difficult to predict and control owing to the generation of multiple siRNAs species with varying $3^{\prime}$ - and $5^{\prime}$-ends, it is difficult to control the degrees of silencing of target genes through dsRNA.

Recently, artificial miRNA (amiRNA) and tasiRNA (atasiRNA) technologies have been developed (Baykal and Zhang 2010; Baulcombe et al. 2006; Schwab et al. 2006; de la Luz et al. 2008; Ossowski et al. 2008). Both of these silencing technologies possess intrinsic advantages over dsRNA-induced silencing approach and overcome limitations including non-specificity of dsRNA. Artificial miRNA and atasiRNA utilize miRNA (19-24nt) and siRNA (21nt) which are complementary to their target sequences and are, therefore, highly sequence-specific and can minimize off-target effect and silence genes sharing a high degree of homology. These short RNAs can be engineered to carry $3^{\prime}$-end mismatches that minimize the transitive silencing due to avoiding RDR activity (Baulcombe 2007; Voinnet 2008). Desirable and varying degrees of silencing of target
mRNAs could be achieved by creating different numbers of mismatches (e.g., 1-3). By enabling highly transcriptspecific knockdown and thus more conclusive results, or by avoiding lethality without generating a relatively large number of RNAi lines, these advanced features will make functional tests of target genes available to plant scientists. Finally, these two new technologies, i.e., amiRNA and atasiRNA, make it possible to use a single promoter to drive a single transgene cassette (avoiding use of multiple expression cassettes) to carry different amiRNA or atasiRNA species to simultaneously silence multiple genes of either same or different pathways for complex metabolic engineering. This is difficult to achieve using dsRNA construct.

However, the design of amiRNA remains complicated because the pre-miRNA secondary structure in amiRNA expression cassette is highly sensitive to a change in the 19- to 24-nt miRNA sequence and thus it is hard to predict the effect of designed miRNA on the pre-miRNA structure and silencing efficiency. Also, cloning of multiple pre-miRNAs into the amiRNA cassette in a construct could be technically challenging and the resulting long-stranded cassette may cause undesirable impacts on amiRNA processing and functions. However, atasiRNA technology could overcome the limitations of the amiRNA approach (Baulcombe et al. 2006; de la Luz et al. 2008). A single copy of atasiRNA carried by a TAS locus will silence the mRNA target (de la Luz et al. 2008). And a single TAS locus can be engineered to contain either multiple atasiRNAs that target different sites of the same gene to enhance silencing efficiency or carry multiple atasiRNAs (each specific to a target mRNA) to simultaneously silence multiple genes in a complex metabolic engineering feat (Baulcombe et al. 2006; de la Luz et al. 2008). Using single TAS locus has a significant advantage over amiRNA technology—as single TAS locus avoids technical challenges in cloning and minimizes possible undesirable impact of long-stranded multiple pre-miRNAs. Moreover, a TAS locus (e.g., TAS1) can be co-introduced and coexpressed with miRNA trigger (e.g., miR173) into any plant species for either constitutive or inducible silencing of target genes (Baulcombe et al. 2006). From the regulatory point of view, using a minimal number of transgene cassettes via atasiRNA strategy in a genetically modified (GM) crop will ease regulatory work and save cost. Figure 2 depicts principles of atasiRNA approach.

Very recently, microRNA-induced gene silencing (MIGS) approach has been developed (Felippes et al. 2012). This new silencing platform is essentially derived from the tasiRNA pathway using miRNA173 as a trigger to generate phased siRNA (Fig. 3). However, each sequence used to silence target mRNA is fused with miRNA173 trigger at $5^{\prime}$ end and can be over hundreds of nucleotides long. Such a long silencing sequence will further ensure efficiency. Nevertheless, MIGS retains the limitation of the 
A

dsRNA pathway

dsRNA

DCL4

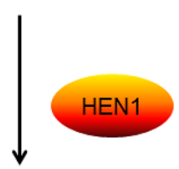

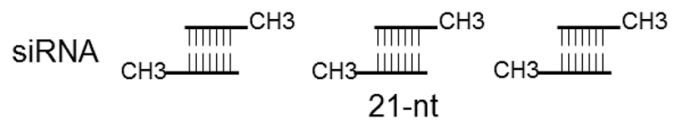
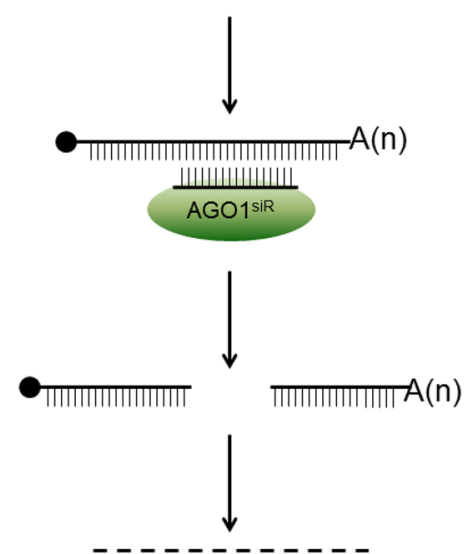

Degradation
B

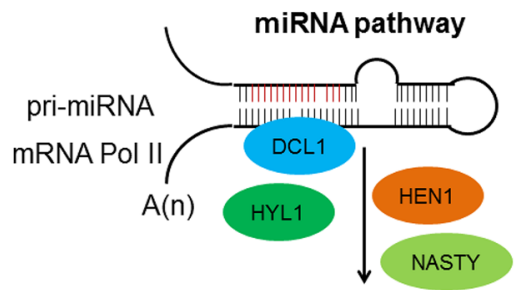

$\mathrm{miR} / \mathrm{miR}^{*}$
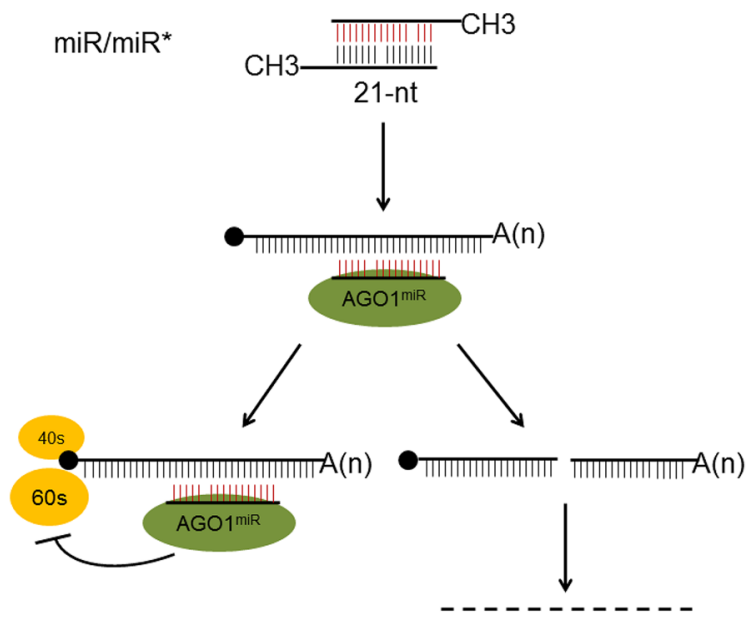

Degradation

C

tasiRNA pathway

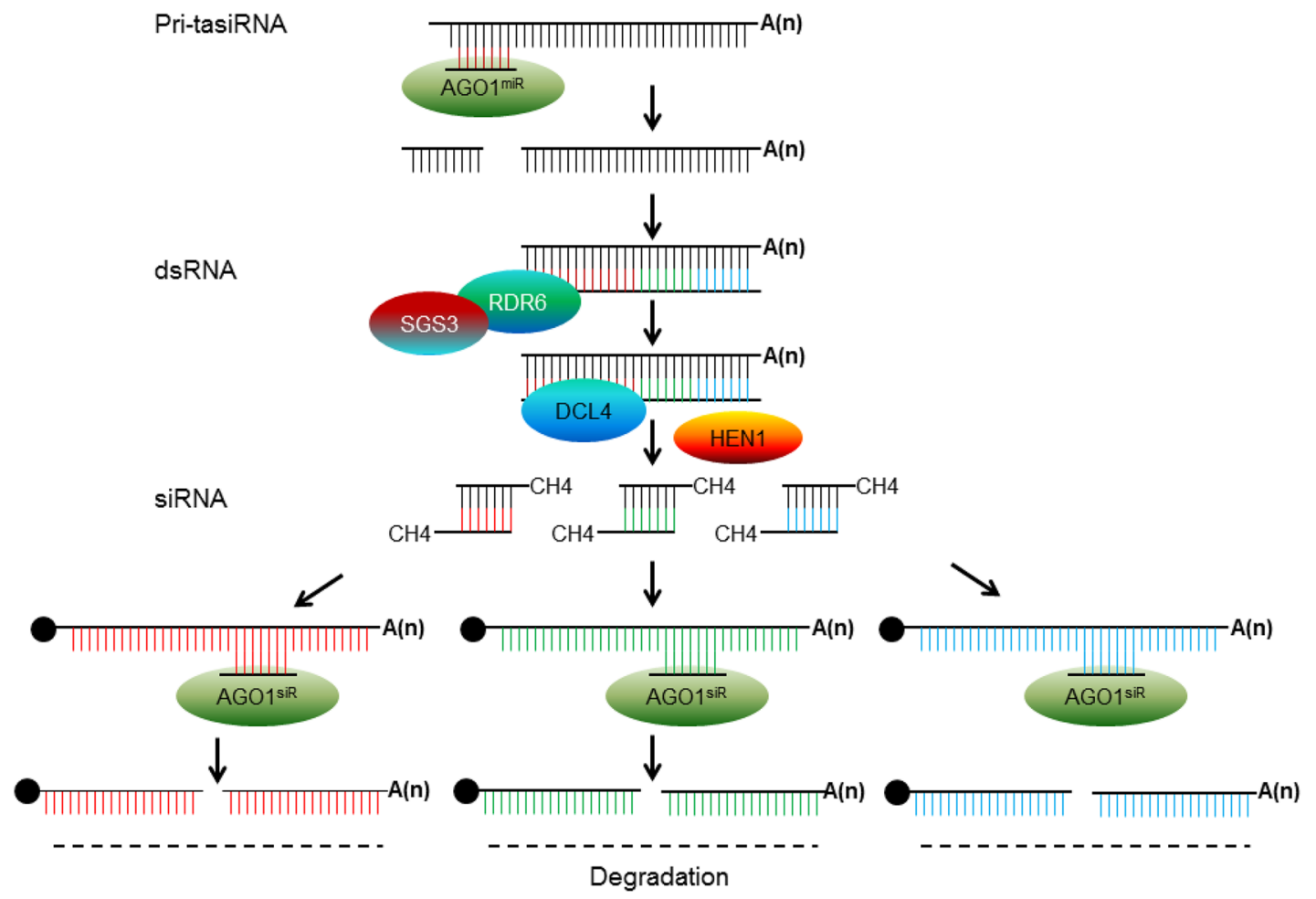


4 Fig. 1 Small regulatory RNA pathways. a Double-stranded (ds) RNA pathway. A long dsRNA with perfect complementary repeats from a transgene construct is diced, by DCL4, to 21-nt siRNA, which is then methylated by HEN1. One of the 21-nt siRNA strands is uploaded to AGO1 in RISC and guides sequence-specific degradation of target RNA. Note: 24-nt siRNA-mediated methylation pathway is not shown. b miRNA pathway. Pri-miRNA transcript of stem-loop structure from a genomic locus is produced through RNA Pol II. Mature miRNA is then generated from pri-mRNA by concerted action of DCL1 and HYL1 and further methylated by HEN1. The mature and methylated miRNA is then exported out of the nucleus, mediated by HASTY, and uploaded to AGO1 in RISC to guide sequence-specific degradation or translational repression of target transcript, or DNA methylation. $\mathbf{c}$ tasiRNA pathway. Pri-tasiRNA transcript is first transcribed from genomic TAS locus, cleaved by miRNA through AGO1, double-stranded through cooperative activity of SGS3 and RDR6, diced to 21-nt siRNA, and methylated by HEN1. The 21-nt siRNA then guides sequence-specific degradation of target transcript. AtasiRNA enables to silence several different target mRNAs simultaneously. Note: nucleotide bonds of different colors indicate different sequences of either siRNAs, miRNAs, or target mRNAs
dsRNA platform, that is, the high chance of causing offtarget effect and transitivity.

Success in atasiRNA necessitates accurate design of mRNA target sites

Because of its intrinsic advantages, atasiRNA is an emerging tool for highly specific and effective gene silencing in plant biology studies and crop improvements. However, it is critical to be able to design the 21-nt atasiRNA accurately to effectively silence mRNA targets. Because a 21-nt atasiRNA binds to a very short stretch of target sequence, the most effective site of a target mRNA needs to be identified. Such a 21-nt atasiRNA is in contrast with the dsRNA from transgene IR which generates multiple siRNAs and thus binds to many different sites across the target mRNA. Although multiple atasiRNAs can be deployed within a

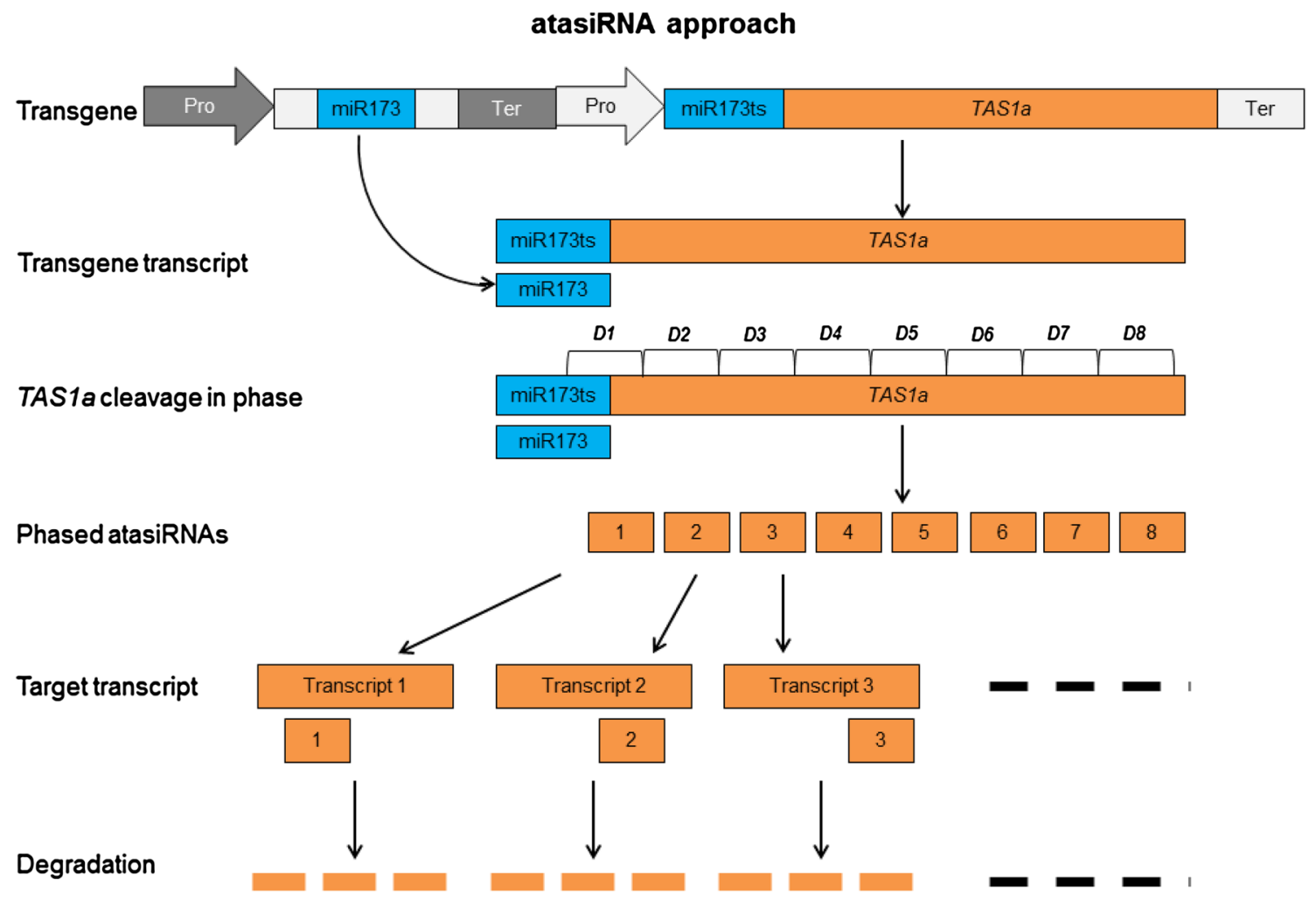

Fig. 2 atasiRNA approach using TASla as an example. Transgene expression cassette is composed of two expression units for overexpressing miR173 and TAS1a, respectively. Each unit has its own promoter and terminator. The miR173 cassette generates miR173 precursor which is then processed to produce mature miR173 that binds to miR173 trigger sequence (miR173ts) at $5^{\prime}$ region of the TASla transcript. Such a binding triggers TASla cleavage in phase, producing phased atasiRNAs. The 21-nt atasiRNA in each phasing position (bracket) can be designed as either siRNA or miRNA. For cassette construction, single atasiRNA can be built into the TAS1a locus using overlapping PCR while TAS1a carrying multiple atasiRNAs can be synthesized. Each atasiRNA can bind to an endogenous transcript, causing either transcript cleavage or translational repression of the target transcript. The scheme shows transcript cleavage only. Note: Pro and Ter: promoter and terminator, respectively. Different colors in promoters and terminators indicate different sequences whereas the numbers mean different phasing positions in TASIa or target transcripts of different sequences. Black dotted lines suggest degradation of more target transcripts. A similar design can be applied to employing TAS1b, TAS1c and TAS2 for atasiRNA platform 
MIGS platform

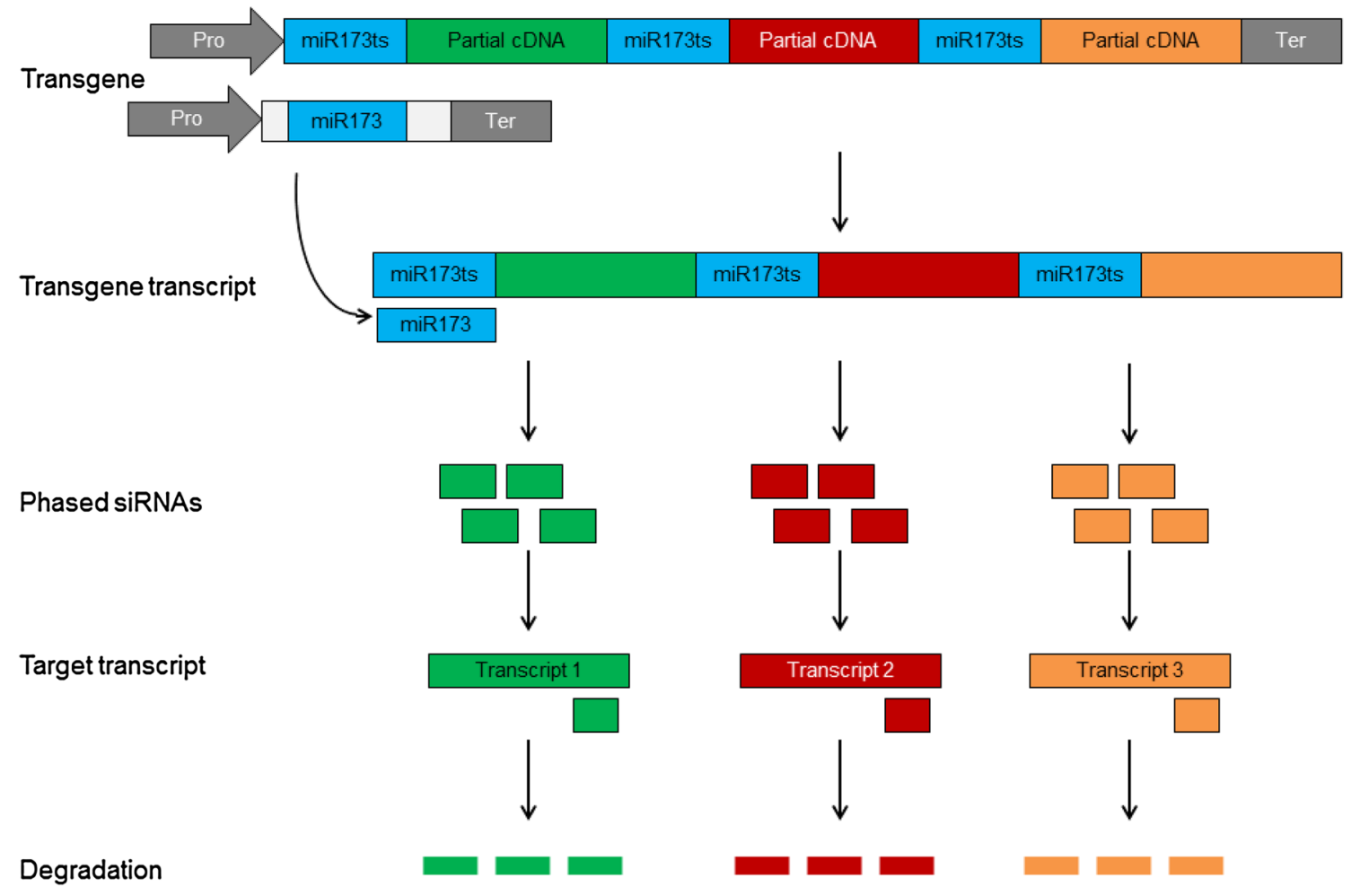

Fig. 3 microRNA-induced gene silencing platform. A transgene cassette includes a single promoter and terminator driving multiple expression units. Each unit carries a miR173 trigger sequence (miR173ts) fused with a partial cDNA at downstream. Each cDNA silences a specific target transcript. These multiple units generate a long transcript including miR173ts and complementary transcript to

TAS locus to bind different sites of an mRNA target to warrant a high degree of silencing, using a single atasiRNA remains more desirable because it provides more specificity and reproducibility of the silencing results (Tilesi et al. 2009). Importantly, a high success rate of atasiRNA prediction will minimize the number of atasiRNA target sites to be screened through in vivo tests in a model plant or in vitro transient assays to silence mRNA targets. Such "pilot" screens are currently necessary before the tasiRNAexpressing cassette can be actually introduced into a crop genome to warrant successful RNAi. Therefore, a precise atasiRNA design to minimize these pilot tests will make RNAi-mediated silencing study and crop trait improvements both cost-effective and time-saving.

Design of effective siRNAs for plant mRNA targets can be substantially improved by employing new approaches

Recently, significant progress has been made in the design and validation of siRNAs or miRNAs for mRNA targets. Of various design programs, WMD3 (http://wmd3.weigelworld.org) each cDNA. Binding of mature miR173 to the miR173ts triggers production of phased 21-nt siRNAs. The siRNAs derived from each partial cDNA will cleave plant endogenous complementary transcripts. Note: Pro and Ter: promoter and terminator, respectively. Different colors indicate different nucleotide sequences

has been widely adapted to aid amiRNA designs to silence plant mRNA targets. In principle, this program can be also used to predict atasiRNAs to be carried by TAS locus to silence mRNA targets. However, there are significant limitations of this design program. It generates a long list of amiRNA candidates for each target gene and score candidates by sequence complementarity and hybridization energy with little consideration of mRNA structure and bounded-regulatory proteins, leading to unknown in vivo efficacy ( $\mathrm{Li}$ et al. 2013).

Very recently, a new breakthrough has been made in validating effective miRNAs by employing epitopetagged protein-based amiRNA screens ( $\mathrm{Li}$ et al. 2013). In this improved approach, a DNA sequence encoding target mRNAs and its adjacent epitope-tagged protein are constitutively or inducibly coexpressed in a transient expression system such as protoplasts with amiRNA candidates targeting single or multiple genes. Because the design principles of amiRNA and atasiRNA candidates are very similar, this new approach can be readily employed to validate atasiRNA (unpublished results), substantially improving the 
effectiveness of the atasiRNA design and enhancing plant biology study and crop improvements.

atasiRNA remains a unique and effective tool as other technologies are emerging

Over the past 12 months, another new tool has emerged and is being further developed for precise genome editing, namely clustered regularly interspaced short palindromic repeats (CRISPR/Cas) ( $\mathrm{Li}$ et al. 2014). This editing platform is from bacterial Type II CRISPR locus and employs non-coding RNA to recognize target genome sequence. One of the key advantages of this new platform is its ability for multiplex genome editing, which other precise genome editing approaches including zinc finger nuclease and transcription activator-like effector nuclease do not possess. This multiplex feature is in some way similar to atasiRNA but the former edits target genome DNA rather than transcript. However, a key advantage of atasiRNA is its ability to minimize off-target effect through the employment of 21-nt miRNA species within TAS locus (unpublished). In addition, different levels of target transcript knockdown can be achieved through atasiRNA which avoids lethality. These two features are significantly advantageous over the above approaches for plant biology study and field application.

\section{Conclusion}

We are entering a new era where new tools are being developed at an accelerated pace. These new tools enhance the analysis and utilization of enormous genomic information coming from post-genome research, particularly, the completion of whole genome sequences in many plant species. Of various tools, atasiRNA plays a uniquely important role in plant biology study and crop genetic improvements.

\section{References}

Allen E, Xie Z, Gustafson AM, Carrington JC (2005) microRNAdirected phasing during trans-acting siRNA biogenesis in plants. Cell 121:207-221

Auer C, Frederick R (2009) Crop improvement using small RNAs: applications and predictive ecological risk assessments. Trends Biotechnol 27:644-651

Baulcombe DC (2007) Molecular biology. Amplified silencing. Science 315:199-200

Baulcombe D, Krebbers E, Hitz W, Glassman KF, Aukerman MJ, Williams R, Yoo BC (2006) Methods and compositions for gene silencing. Pioneer Hybrid International, Inc. and E.I. DuPont De Nemours and Company. PCT/US2006/023607

Baykal U, Zhang Z (2010) Small RNA-mediated gene silencing for plant biotechnology. In: Catalano AJ (ed) Gene silencing: theory, techniques and applications. Nova Pub, New York, pp 255-269
Bellés X (2010) Beyond Drosophila: RNAi in vivo and functional genomics in insects. Annu Rev Entomol 55:111-128

Bevan MW, Uauy C (2013) Genomics reveals new landscapes for crop improvement. Genome Biol 14:206

Bonfim K, Faria JC, Nogueira EO, Mendes EA, Aragão FJ (2007) RNAi-mediated resistance to Bean golden mosaic virus in genetically engineered common bean (Phaseolus vulgaris). Mol PlantMicrobe Interact 20:717-726

Brodersen P, Voinnet O (2006) The diversity of RNA silencing pathways in plants. Trends Genet 22:268-280

Brodersen P, Sakvarelidze-Achard L, Bruun-Rasmussen M, Dunoyer P, Yamamoto YY, Sieburth L, Voinnet O (2008) Widespread translational inhibition by plant miRNAs and siRNAs. Science 320:1185-1190

Chapman EJ, Carrington JC (2007) Specialization and evolution of endogenous small RNA pathways. Nat Rev Genet 8:884-896

Chen X (2009) Small RNAs and their roles in plant development. Annu Rev Cell Dev Biol 25:21-44

de la Luz Gutiérrez-Nava M, Aukerman MJ, Sakai H, Tingey SV, Williams RW (2008) Artificial trans-acting siRNAs confer consistent and effective gene silencing. Plant Physiol 147:543-551

Dodo HW, Konan KN, Chen FC, Egnin M, Viquez OM (2008) Alleviating peanut allergy using genetic engineering: the silencing of the immunodominant allergen Ara $\mathrm{h} 2$ leads to its significant reduction and a decrease in peanut allergenicity. Plant Biotechnol J 6:135-145

Dunoyer P, Schott G, Himber C, Meyer D, Takeda A, Carrington JC, Voinnet O (2010) Small RNA duplexes function as mobile silencing signals between plant cells. Science 328:912-916

Eady CC, Kamoi T, Kato M, Porter NG, Davis S, Shaw M, Kamoi A, Imai S (2008) Silencing onion lachrymatory factor synthase causes a significant change in the sulfur secondary metabolite profile. Plant Physiol 147:2096-2106

Felippes FF, Wang JW, Weigel D (2012) MIGS: miRNA-induced gene silencing. Plant J 70:541-547

Feuillet C, Leach JE, Rogers J, Schnable PS, Eversole K (2011) Crop genome sequencing: lessons and rationales. Trends Plant Sci 16:77-88

Fire A, Xu S, Montgomery MK, Kostas SA, Driver SE, Mello CC (1998) Potent and specific genetic interference by doublestranded RNA in Caenorhabditis elegans. Nature 391:806-811

Flores T, Karpova O, Su X, Zeng P, Bilyeu K, Sleper D, Nguyen H, Zhang Z (2008) Silencing of GmFAD3 gene by siRNA leads to low $\alpha$-linolenic acids (18:3) of fad3-mutant phenotype in soybean [Glycine max (Merr.)]. Transgenic Res 17:839-850

Gasciolli V, Mallory AC, Bartel DP, Vaucheret H (2005) Partially redundant functions of Arabidopsis DICER-like enzymes and a role for DCL4 in producing trans-acting siRNAs. Curr Biol 15:1494-1500

Gilissen LJ, Bolhaar ST, Matos CI, Rouwendal GJ, Boone MJ, Krens FA, Zuidmeer L, Van Leeuwen A, Akkerdaas J, Hoffmann-Sommergruber K, Knulst AC, Bosch D, Van de Weg WE, Van Ree R (2005) Silencing the major apple allergen Mal d 1 by using the RNA interference approach. J Allergy Clin Immun 115:364-369

Jackson AL, Bartz SR, Schelter J, Kobayashi SV, Burchard J, Mao M, Li B, Cavet G, Linsley PS (2003) Expression profiling reveals off-target gene regulation by RNAi. Nature Biotechnol 21:635-937

Jones-Rhoades MW, Bartel DP, Bartel B (2006) microRNAs and their regulatory roles in plants. Ann Rev Plant Biol 57:19-53

Kumar P, Kamle M, Pandey A (2012) RNAi: new era of functional genomics for crop improvement. In: Goyal A, Maheshwari P (ed) Frontiers on recent developments in plant science, vol 1. Bentham Sci Pub, pp 24-38

Le L, Lorenz Y, Scheurer S, Fötisch K, Enrique E, Bartra J, Biemelt S, Vieths S, Sonnewald U (2006) Design of tomato fruits with 
reduced allergenicity by dsRNAi-mediated inhibition of ns- LTP (Lyc e 3) expression. Plant Biotechnol J 4:231-242

Li JF, Chung HS, Niu Y, Bush J, McCormack M, Sheen J (2013) Comprehensive protein-based artificial microRNA screens for effective gene silencing in plants. Plant Cell 25:1507-1522

Li JF, Zhang D, Sheen J (2014) Epitope-tagged protein-based artificial microRNA screens for optimized gene silencing in plants. Nat Protoc (in press)

Liu Q, Singh SP, Green AG (2002) High-stearic and high-oleic cottonseed oils produced by hairpin RNA-mediated post-transcriptional gene silencing. Plant Physiol 129:1732-1743

Martienssen R (2010) Small RNA makes its move. Science 328:834-835

Meyer P, Saedler H (1996) Homology-dependent gene silencing in plants. Annu Rev Plant Physiol Plant Mol Biol 47:23-48

Molnar A, Melnyk CW, Bassett A, Hardcastle TJ, Dunn R, Baulcombe DC (2010) Small silencing RNAs in plants are mobile and direct epigenetic modification in recipient cells. Science 328:872

Ogita S, Uefuji H, Yamaguchi Y, Koizumi N, Sano H (2003) Producing decaffeinated coffee plants. Nature 423:823

Ossowski S, Schwab R, Weigel D (2008) Gene silencing in plants using artificial micro RNAs and other small RNAs. Plant J 53:674-690

Page DR, Grossniklaus U (2002) The art and screen of genetics: Arabidopsis thaliana. Nautre 3:124-136

Peragine A, Yoshikawa M, Wu G, Albrecht HL, Poethig RS (2004) SGS3 and SGS2/SDE1/RDR6 are required for juvenile development and the production of trans-acting siRNAs in Arabidopsis. Genes Dev 18:2368-2379

Rajagopalan R, Vaucheret H, Trejo J, Bartel DP (2006) A diverse and evolutionarily fluid set of microRNAs in Arabidopsis thaliana. Genes Dev 20:3407-3425

Schwab R, Ossowski S, Riester M, Warthmann N, Weigel D (2006) Highly specific gene silencing by artificial microRNAs in Arabidopsis. Plant Cell 18:1121-1133

Shafran H, Miyara I, Eshed R, Prusky D, Sherman A (2008) Development of new tools for studying gene function in fungi based on the Gateway system. Fungal Genet Biol 45:1147-1154
Shi J, Wang H, Schellin K, Li B, Faller M, Stoop JM, Meeley RB, Ertl DS, Ranch JP, Glassman K (2007) Embryo-specific silencing of a transporter reduces phytic acid content of maize and soybean seeds. Nature Biotechnol 25:930-937

Shimizu T, Yoshii M, Wei T, Hirochika H, Omura T (2009) Silencing by RNAi of the gene for Pns12, a viroplasm matrix protein of Rice dwarf virus, results in strong resistance of transgenic rice plants to the virus. Plant Biotechnol J 7:24-32

Siomi H, Siomi MC (2009) On the road to reading the RNA-interference code. Nature 457:396-404

Tilesi F, Fradiani P, Socci V, Willems D, Ascenzioni F (2009) Design and validation of siRNAs and shRNAs. Curr Opin Mol Ther $11: 156-164$

Vaucheret H (2006) Post-transcriptional small RNA pathways in plants: mechanisms and regulations. Gene Dev 20:759-771

Vazquez F, Vaucheret H, Rajagopalan R, Lepers C, Gasciolli V, Mallory AC, Hilbert JL, Bartel DP, Crété P (2004) Endogenous transacting siRNAs regulate the accumulation of Arabidopsis mRNAs. Mol Cell 16:69-79

Voinnet O (2008) Use, tolerance and avoidance of amplified RNA silencing by plants. Trends Plant Sci 13:317-328

Waterhouse PM, Helliwell CA (2003) Exploring plant genomes by RNA-induced gene silencing. Nature Rev Genet 4:29-38

Watson JM, Fusaro AF, Wang M, Waterhouse PM (2005) RNA silencing platforms in plants. FEBS Lett 579:5982-5987

Xie Z, Allen E, Wilken A, Carrington JC (2005) DICER-LIKE 4 functions in trans-acting small interfering RNA biogenesis and vegetative phase change in Arabidopsis thaliana. Proc Natl Acad Sci USA 102:12984-12989

Yin X, Zhang Z (2010) Soybean functional genomics through reverse genetics: opportunities and challenges. In: Columbus F (ed) Functional genomics. Nova Sci Pub, New York, pp 225-236

Yoshikawa M, Peragine A, Park MY, Poethig RS (2005) A pathway for the biogenesis of trans-acting siRNAs in Arabidopsis. Genes Dev 19:2164-2175 